Abstract

\title{
Characterising Interactions between Influenza A Virus and Respiratory Syncytial Virus during In Vitro Coinfection ${ }^{+}$
}

\author{
Joanne Haney *, Kieran Dee, Colin Loney, Swetha Vijayakrishnan and Pablo R. Murcia \\ MRC-University of Glasgow Centre for Virus Research, Glasgow G61 1QH, UK; \\ kieran.dee@glasgow.ac.uk (K.D.); colin.loney@glasgow.ac.uk (C.L.); \\ swetha.vijayakrishnan@glasgow.ac.uk (S.V.); pablo.murcia@glasgow.ac.uk (P.R.M.) \\ * Correspondence: j.haney.1@research.gla.ac.uk \\ + Presented at Viruses 2020-Novel Concepts in Virology, Barcelona, Spain, 5-7 February 2020.
}

Published: 14 June 2020

\begin{abstract}
Influenza A virus (IAV) and respiratory syncytial virus (RSV) are important respiratory pathogens that share common epidemiological features and cellular tropism within the respiratory tract. This gives rise to the potential for biological interactions between IAV and RSV during coinfection of hosts. Virus-virus interactions are increasingly recognised for their contribution to viral dynamics during infection, however, the molecular processes underpinning these interactions are unknown. Here, we developed an in vitro coinfection system to characterise the infection dynamics of IAV (A/Puerto Rico/8/34, H1N1) and RSV (A2) in single virus infection or coinfection in lung epithelial cells, with the aim to identify biological processes that drive virus-virus interactions during coinfection. We compared viral replication kinetics at different multiplicities of infection and observed that RSV replication was inhibited during coinfection with IAV, whilst IAV replication was facilitated by coinfection. To further characterise IAV/RSV interactions, we determined the relative proportions of single virus infected or coinfected cells during early and late timepoints post-infection and observed differences in expression of viral proteins between single and coinfected states. Additionally, cell viability was measured determine differences in viralinduced cytopathic effect. Compared with RSV infection, cell death is induced at earlier timepoints post IAV infection and coinfection, indicating that different cellular processes are initiated in response to infection. These studies highlight that both competitive and facilitative ecological interactions occur between IAV and RSV during coinfection and shed light on sources of potential interactions at the cellular and molecular level.
\end{abstract}

Keywords: coinfection; influenza A virus; respiratory syncytial virus; virus-virus interactions

Funding: This research was funded by the UK Medical Research Council grant number MR/N013166/1.

(C) 2020 by the authors. Licensee MDPI, Basel, Switzerland. This article is an open access article distributed under the terms and conditions of the Creative Commons Attribution (CC BY) license (http://creativecommons.org/licenses/by/4.0/). 